\title{
The role of CAS concepts and methods in EE education and professional activities: societal challenges and lessons learned from Alfred Fettweis
}

\author{
Joos Vandewalle, Fellow, IEEE, ${ }^{1}$
}

\begin{abstract}
Evolutions in technology and society and in particular the grand challenges of engineering require a proactive strategy and actions for $C A S$ research and education. Indeed, these have a direct impact on the outcome of CAS education in terms of our EE graduates and on the outcome of $C A S$ research and professional activities in terms of designs of devices, systems, services and deeper findings. Our students should have a growth mindset with skills and insights that support an evolving professional horizon of at least 40 years. The paper builds on the contributions and insights of Alfred Fettweis and other new insights in order to produce interesting avenues for dealing with the challenges in the areas of interest within the scope of the circuits and systems society. In particular the role of mathematics in EE education and research are highlighted.
\end{abstract}

Index Terms-circuits and systems, engineering education, mathematics, systems engineering education

\section{INTRODUCTION}

It has been widely observed around the globe that our recent student generations are different from the older generations and in particular that their attention span is getting shorter [1,2]. Related to CAS education, Tsividis [3], [4] had attracted our attention to these phenomena already some time ago, and the recent advent of social media and smartphones has definitely exaggerated this phenomenon. In addition, several technologies become obsolete in the evolving society or are unable to solve the challenges of our society, and hence new technologies should emerge [5]. In this context, this paper aims at a fresh reflection on the role of mathematics in circuits and systems research and education and in approaches that can work in this context. In the process one can learn several lessons from Prof. Alfred Fettweis, and from more recent deep insights and pedagogic innovations of Jo Boaler and Carol Dweck [6-8] on the mathematical growth mindset.

In Section II the role of mathematical abstractions and the importance and benefits of a growth mindset for students in mathematics as well as in EE are presented. Moreover, these two pedagogic approaches are mutually reinforcing. Section III focusses on the system concepts and their role in EE education and professional activities. The important links with societal issues and global engineering challenges are presented in Section IV. Finally, the last section V presents conclusions and recommendations.

II. THE ROLE OF ABSTRACTION AND THE BENEFITS OF A GROWTH MINDSET FOR EDUCATION IN MATHEMATICS AND CAS AND HOW THESE ARE LINKED TO PROFESSIONAL ENGINEERING

\section{ACTIVITY}

As mentioned before, the context of technology and society has sufficiently changed over the past 25 years. Hence it is a good exercise to question and rethink the goals and methods of engineering education. The increase of complexity of technology requires an increase in abstraction to still be able to handle complex systems. Modern Mathematics could be a candidate subject, that provides the necessary tools and insights to achieve this abstraction. In this section we will advocate an approach that is based on the lessons learned from Professor Fettweis and from recent findings on mathematics education. Mathematics supported by physical intuition was a crucial element for the entire career and many achievements of Prof. Fettweis. One can refer here to a very interesting orally recorded interview, that is also transcribed and can be downloaded from the Engineering and Technology History Wiki [9]. Interviewer Nebeker: "Well, it is the strong mathematics background that ties all these things together." Fettweis: "It's all mathematics, and the broad educational background."

Let us illustrate this with the invention of the switched

\footnotetext{
date of submission of the paper for review, July 17, 2018.

J. Vandewalle is with Electrical Engineering Department ESAT/Stadius of the Katholieke Universiteit Leuven, Belgium, (e-mail: Joos.Vandewalle@esat.kuleuven.be).
} 
capacitor circuits. This is a notorious example of his visionary innovations, based on deep mathematical insights, that anticipated on implementation, and product innovation. During his doctorate period at our sister university UCL in the same location of city of Leuven in 1963 he investigated the design of passive filters. The traditional way of designing passive filters at that time was the use of LC ladder networks, and there is extensive research activity and a collection of publications in the years before 1960 on these methods. In his thesis he demonstrated mathematically that it is possible to construct filters by using only capacitors and periodically controlled switches thereby avoiding the use of costly inductances [10]. At that time, such switches were difficult to realize, and hence his finding was dormant as a mathematical curiosity. Fourteen years later, due to progress in the semiconductor technology, cheap MOS transistor switches could be fabricated and the theory of Fettweis was, to a certain extent, reinvented in the US as switched capacitor circuits. Today, every electronic switchboard contains tens of thousands of such filters in chip technology.

Hence Fettweis advocated ${ }^{2}$ that EE education should excite mathematical insights in the students. Mathematics is for him not only a tool but a formation of mind [11]. Mathematical research typically produces new branches in a growing mathematics tree, often away from engineering and often continually becoming more abstract and formalistic. On the other hand, problems posed by society, technology, ICT, digitization require new generations of mathematically oriented engineers to create new targeted mathematical developments "We must, wherever possible, develop mathematical theories that sacrifice generality for the sake of simplicity, but without sacrificing rigour" [11]. This is very much in line with inspirational approaches to simplify mathematics teaching as presented in the "street-fighting mathematics" [12]. The book is a rich source of intuitive, practical, and attractive shortcuts, tricks, and brilliant mathematical techniques, that avoid lengthy mathematical proofs and derivations, that are quite precise, but most often boring and not intuitive. The six tools are dimensional analysis, attention on easy cases, lumping, picture proofs, successive approximation, and reasoning by analogy. Here again we see that this is in line with the vision of Fettweis when he is addressing the issue of producing alternative proofs of mathematical theorems for engineering students [11]: " For these reasons, it is obligatory that we learn to value properly activities in engineering science that aim at substantially improving the presentation of existing knowledge."

This can be a stimulating exercise for CAS education. Some examples are described in a case study of the Thévenin/Helmholtz and Norton/Mayer equivalents [13], where the focal concept is a straight line in the i-v plane, and others can be found in $[14,15]$. And indeed "Our brain is more developed visually than symbolically". Fettweis advocates attractive mathematics, close to the physics. Mathematics is a constructive mental activity. Mathematics is the natural language for EE. During his EE education he was deeply immersed in mathematics. Moreover, he advocated [9] the importance of an entrance examination on mathematics for engineering in Belgium "That is the reason why so many Belgian engineers are so strongly mathematically oriented, or why often the students most gifted in mathematics go into engineering". And "There are quite a few Belgians among the mathematically oriented scientists in the IEEE because of this inclination towards mathematics. I am myself very mathematically oriented. So, I can be part of the proof of this."

Teaching in engineering should not only introduce the students in the present state of the art, but also be ahead of the currently available computer power and technology as a whole and provide the basics that can support and stimulate a professional career of 40 years (Figure 1).

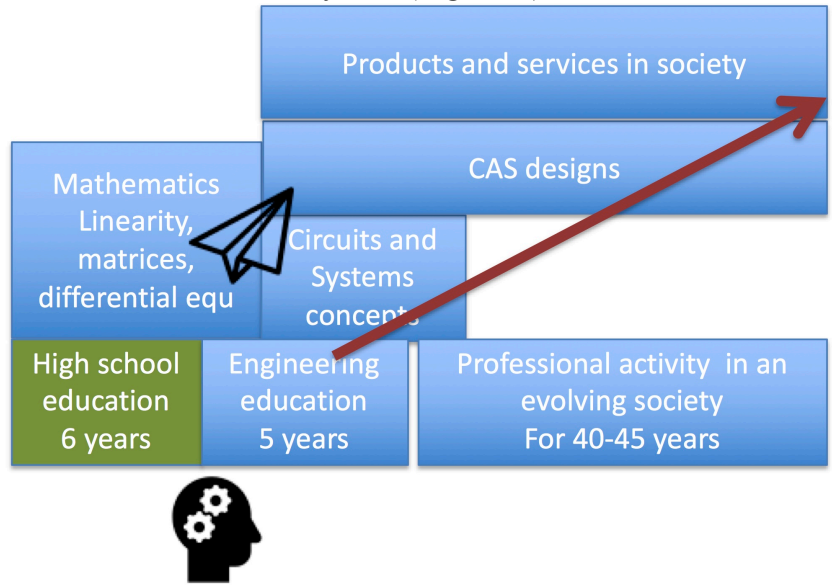

Fig. 1 High school and university education provide concepts, insights, and wisdom on which a professional career is developed. Hence there is a career trajectory for EE from concepts, via circuits and systems towards products and services.

Hence the educational trajectory of 6 years of high school and 5 years of engineering education should target a much longer period. So, the concepts and the methods that are taught in engineering should not only serve the immediate use when starting professionally, but also the later phases. The author was teaching many years linear algebra and basic circuits and system theory to first and second year engineering students. It often happened that topics of these courses like singular value decomposition of reasonably sized matrices, that occur in practice, were impossible to be computed with the computational power at the time of teaching. However, after 5 years of engineering education the computer power of up-todate computers had increased by a factor of 10 . Hence the students could build in their professional activities on such more advanced topics. This was also nicely formulated in the obituary for Alfred Fettweis [16] in the CAS newsletter: "Like most great men in art and science, he combined an immense interdisciplinary wisdom, natural intelligence for the creation of basic concepts and the enthusiasm for teaching these to the

\footnotetext{
${ }^{2}$ cfr lecture of A. Fettweis in 1989 at the celebration of 125 years of engineering education at KU Leuven
} 
next generation. During his years in industry he experienced from inside that visionary basic engineering science does exist and can in the long run lead to great economic returns."

Quite often students in high school as well as at universities are traumatized by math. When they get the idea that they cannot do mathematics, most often they keep these negative feelings towards math for the rest of their life. Since engineering and electrical engineering in particular strongly build on mathematical concepts and skills, these negative feelings are often also present towards engineering. In fact, it is widely accepted that these negative feelings and the rude comments in the media reduce the number of entering engineering students in many countries, so that there is a widespread and rather permanent shortage of engineers.

In this context, the research work of Carol Dweck brings radically new insights as well as teaching methods based on the power of the mindset. Building on this work of Dweck, Jo Boaler concretised these insights and approaches for the education of mathematics [6]: "People with a growth mindset are those who believe that smartness increases with hard work, whereas those with a fixed mindset believe that you can learn things, but you can't change your basic level of intelligence". She formulated and debunked 6 widespread myths: (1) Some people can do math and others just can't. (2) Memorizing is important for math. (3) Making mistakes is bad and should be discouraged. (4) Speed is crucial in math. (5) Formal elements are crucial in math. (6) For math cooperation has no added value.

Clearly there are also teachers in engineering that still adhere to many of these myths. Extensive research by Jo Boaler and practice with children of different ages proved that these myths are wrong. At a deeper level, she was able to prove by brain research and practice that the memorizers are the least performant in math, that speed is not crucial for math, that everybody can learn math, that you proceed faster when you make mistakes, and that cooperation is stimulating for math. Math should be about patterns, ideas, relationships and visualization and less about formulas and calculations. Students with a fixed mindset generally make less progress. In fact, lowly performing students with a fixed mindset have a less creative and more narrow view on mathematical problems, than those with a growth mindset. Even high performing students with a fixed mindset are often blocked in their creativity for fear of making mistakes, whereas those with a growth mindset produce more courageous and creative out of the box ideas. Even when these ideas are not entirely correct and when the mistake is corrected, the impact on the brain is deeper and more permanent. So, there is a benefit in making mistakes for all students. Also, there should be no memory questions in math exams. A very recent study [7] concentrates on the important role of the training of mathematics teachers to prepare their teaching with growth mindset. The study proved that $5^{\text {th }}$ grade teachers who took the training class achieved significantly higher test results for their students, than a control group of teachers in the same schools who did not take the training. Very remarkably these changes were particularly strong for girls, language learners and economically disadvantaged students. The training of the teachers had direct impact on the self-image of the teachers and the myths that only some people can learn math. When they developed a growth mindset about themselves, they were able to teach students with a mathematical growth mindset approach. These deep insights and bold statements need to be taken serious and also have direct impact on CAS education. There is certainly an important issue among teachers of CAS whether they have a growth mindset, and also teach their students with the model of a growth mindset in the brain of their students.

\section{The DeEp Role of System Theory CONCEPTS}

Whether it is the power grid, the telephone network, the chip of a microprocessor, or a mobile phone, all these devices and systems are some of the most complex engineering systems that affect our daily life. Yet, the basic circuits and systems methods that are crucial for their specification, design, construction, and maintenance are mostly hidden or considered to be self-evident to the wider public. The list of basic concepts includes: power consumption, short circuit and open circuit, current in a capacitor, Kirchhoff Voltage Law (KVL), Kirchhoff Current Law (KCL), Thevenin theorem, Norton theorem, Tellegen's theorem, energy and passivity, graph theory, circuit analysis, filter design, and impedances and hybrid parameters. Furthermore, basic CAS courses have a central role in the electrical engineering education (figure 2).

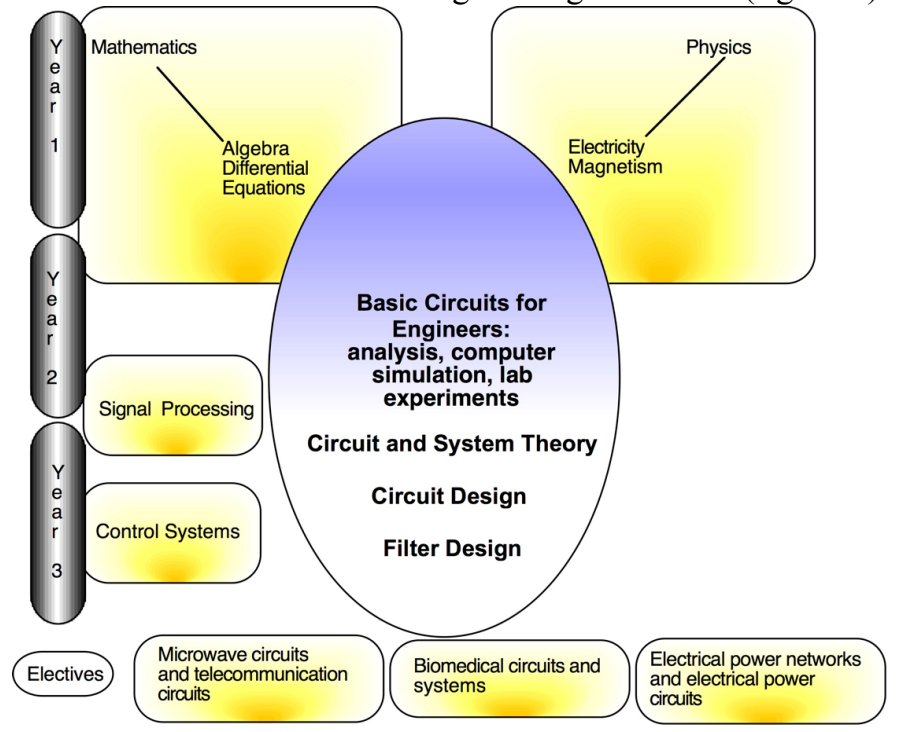

Fig. 2 The central role of circuits and systems courses in electrical engineering education. Links between circuits and systems courses with related courses in electrical engineering.

Continuous and discrete time systems require concepts of signals and systems like impulse response, transfer function, stability, poles, zeros, time constants, Bode diagrams, and interconnections. Basic CAS courses should introduce these concepts in an attractive and simple way, while still maintaining the correctness as illustrated in Figure 3. 


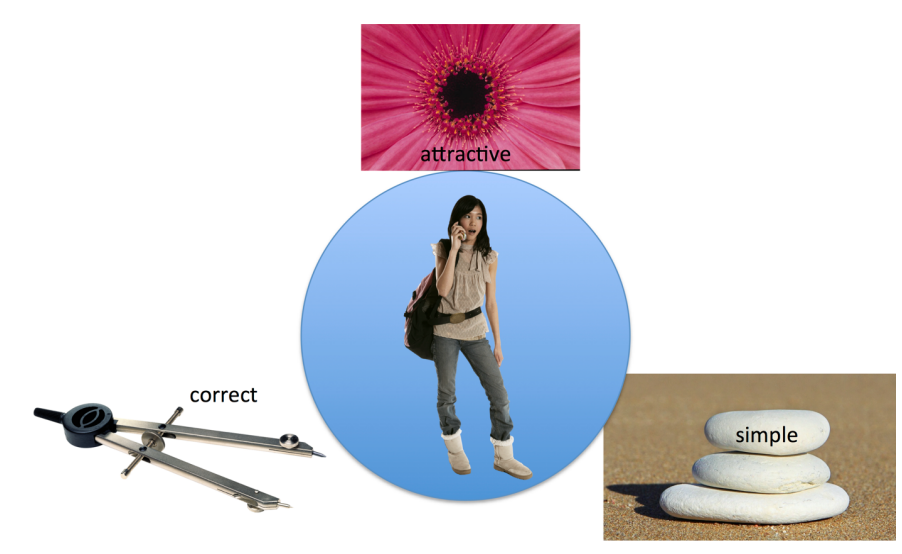

Fig. 3 Issues to be balanced in CAS courses

Extensive experience has also shown that students tend to forget concepts soon after the lectures. Here again it is essential to work with a growth mindset. Moreover, there are often misconceptions around these systems concepts. As mentioned before clarifications $[14,15]$ can consolidate these concepts and produce a deeper imprint in their brain. Also, soon after their introduction during lectures the precision of the insights can be enhanced by feedback through questionnaires, group discussions, exercises, and laboratory sessions, and design projects. Only after such a consolidation phase, one may expect that the concepts are deeply understood and mastered by the students.

There are still deeper system theory concepts that are crucial in EE like stability, sensitivity, robustness, limit cycle behavior, dynamic range, losslessness and passivity. Clearly these are properties that are not dependent on a single component or subsystem but are global and inherent to the complete system. These concepts build on a deeper degree of mathematical abstraction, yet they affect the practical performance of the system profoundly. Of course, software tools like Matlab or Spice can avoid that students should use hand calculations in order to study these properties for their circuit or system designs. Moreover, these tools can deliver useful and attractive plots and visual information. However, the deeper understanding, and the deeper relations among these system characteristics escapes the software tools. And this is nevertheless crucial for students and professionals.

A deep mathematical CAS insight on low-sensitivity based on losslessness, called the Fettweis-Orchard Theorem, was formulated by Alfred_Fettweis [9]: "Anyhow, I realized that this sensitivity problem was related to basic loss. Zero loss in a passive circuit is something you can never go below, because it would mean you have an active device. If all components are passive, the effective loss can never become negative. You need amplification to get a negative effective loss. So, if you have passive devices, at any frequency where the loss reaches zero, that's rock bottom. That means, mathematically speaking, that the sensitivity is zero. If you change a component, if you lower it or make it larger, you cannot get below that value, and therefore the derivative is zero, so you have zero sensitivity.
That is a marvelous property of a filter. It's astonishing that filter designers had not been aware of this, because that property is absolutely fundamental, in my opinion, to the development of carrier telephony, which is essentially a filter-based approach. The fact that we have been able to use such beautiful, sharp filters is essentially due to this sensitivity property. This means that if you design a filter to be a nice filter, you are automatically very insensitive to the parameter influences. It is a fantastic property. Suppose you have a filter of degree ten, you may have in that filter, let's say, twenty components. With a filter of degree ten, you can have zero loss at five different frequencies in the pass-band. Now, the sensitivity at any of these five frequencies is zero, and this with respect to any of the parameters. You have twenty parameters times five, that is $a$ hundred conditions you impose on the sensitivities to be zero. Now you have only twenty parameters in your circuit; how can you satisfy a hundred conditions? In addition, you don't want to waste your freedom completely for getting good sensitivity coefficient; you want to use it to get a good filter curve. Now, the amazing thing is, you get the good sensitivity free of charge. Just design the filter to be a good filter, and these one hundred conditions are automatically fulfilled. That is a fantastic property of passive circuits."

Next at the end of the sixties, when computers were still a curiosity, Fettweis foresaw that the accuracy and reliability of today's communication systems could only be achieved through digitalization. Sound and picture should be represented by numbers, which should be processed by billions of calculations per second. By the way, this evolution is still going on and it is affecting society more and more. Then he conceived the wave digital filters (WDF) that could numerically exhibit the same robustness and quality as the classical passive LC ladder filters. The challenge was to design mathematical algorithms with a limited number of operations, that have as good or better properties than the classical analog filters. The mathematical formulation of the losslessness and passivity properties of doubly terminated LC ladder networks guided him to invent the so-called wave digital filters. This is another illustration that the language of mathematics and a deep insight in the systems behavior is crucial for such impressive new designs. An extension of WDF with the concept of orthogonal filtering is given in another paper [17] of this special issue.

In fact, the move from analog signal processing towards digital signal processing is still continuing. This growing importance of DSP stimulates many EE programs to start with discrete time signal processing and DSP and afterwards turn to continuous time signal processing. Concepts of impulse response and transfer function are clearly easier to understand in discrete time for bachelor EE students, and can provide a stepping stone for the later study of the continuous time counterparts.

\section{EMPHASIzING THE Societal Role of ENGINEERING}

Alfred Fettweis strongly advocated that engineers have a serious responsibility towards society. In 1992 he wrote [11]: "Engineers must be more than ever aware of their responsibility 
towards society. At the same time, society must come to accept that the presence of technology and its influence on our everyday life will continue to expand rapidly in the foreseeable future. Our present question is: what consequences does this have for engineering education? "In the present context one can state that engineering education and profession should include societal issues as they are linked to the grand engineering challenges expressed by the National Academy of Engineering [18]. This view is in line with the vision of CAS Society [19]: "IEEE Circuits and Systems Society (CASS) believe that the Grand Engineering Challenges of the 21 st century can only be addressed in an inter-disciplinary and cross-disciplinary manner. The Society's unique and profound expertise in Circuits, Systems, Signals, Modeling, Analysis, and Design can have a decisive impact on important issues such as Sustainable Energy, Bio-Health, Green Information Technology, NanoTechnology, and Scalable Information Technology Systems." Fettweis uses this argument to advocate [11] an EE education with a deep understanding of the scientific principles "How can we expect a layman to have confidence in, say, a complicated electronic device that controls a potentially dangerous mechanism if the engineer himself does not fully master this device? We cannot expect trust, if the engineer himself does not fully understand what he is doing. We thus arrive at a conclusion similar to the one mentioned previously: we must stress to our students that their responsibility towards society obliges them to make the utmost effort to arrive at an understanding of the scientific principles behind their field, and it is our duty to make these principles available to our students in a way that is as lucid and as accurate as possible."

Whereas pure sciences observe and study nature without interference in nature, engineering intervenes actively in society and nature in this sense that it designs and builds artifacts and services for practical use in society and to improve life. Hence engineering must incorporate the societal consequences of their designs already during the design phase. This has impact as well in research, education as in industrial practice. EE education should stimulate the students to become leaders that tackle in their professional life the 21 Century's Grand challenges for engineering as formulated by the National Academy of Engineering [18]: make solar energy affordable, provide energy from fusion, develop carbon sequestration methods, manage the nitrogen cycle, provide access to clean water, restore and improve urban infrastructure, advance health informatics, engineer better medicines, reverse-engineer the brain, prevent nuclear terror, secure cyberspace, enhance virtual reality, advance personalized learning, engineer the tools for scientific discovery. Several of these challenges have a direct bearing on CAS topics. Moreover, every course in EE should include the societal and ethical aspects related to the topics of the course. As a first example one can mention here the recycling of discarded electronic equipment like smart phones, TV-sets, computers in order to avoid electronic waste [20]. Recycling of this electronic equipment can happen at different levels. First of all, refurbishing and reuse is the best way of recycling and should be facilitated from the design phase on. Next reuse of the metals and valuable materials requires simple dismounting of the electronic circuits and printed circuits. Again, that should be facilitated already during the design and production phase. More generally, the widespread use of social media, internet (of things), and the big data and deep learning brings many benefits in society, but strongly affects the privacy of the users. Since May 25, 2018 GDPR [21] regulates the privacy issues at the European level and forces companies and institutions to treat the personal data respectfully [22]. Hence the designers of ICT and IoT devices must make use of technologies that maintain privacy and allow transparency for the end user. They need to work on 'privacy by design', taking privacy from the start of the design as an important requirement, and not being "stuck" afterwards. The service providers must allow users to assemble services of different origins. The designers of algorithms must write their algorithms to ensure users' privacy. Application designers need to allow transparency, work on efficient and effective technologies that allow users to authorize selectively the use of their private data. Finally, an independent authority has to certify the applications so that users can be confident that the applications are safe. Typically, privacy must be the default option. More examples are given in the conference proceedings of Engineering4Society [23].

This fits nicely with the Systems Engineering (SE) approach. In view of the complexity of many systems SE aims at optimal functioning of the whole system during its entire lifecycle. Hence this ranges from the design \& manufacturing, also to use and disposal. Hence systems engineering encompasses activities involved in the design, development, production and application of machines and instruments in the societal context. More generally, all engineering study programs should include skills of social consciousness, viable entrepreneurship, multidisciplinarity, multicultural understanding, research creativity.

This link with society is also crucial in the ROSE (the Relevance of Science Education) project [24] by Sjoberg and Schreiner on the views of young learners (age 15) for science and technology in 40 countries. Clearly the motivation for Science and Technology at the age of 15 in most developed countries is very low. At that age they are not aware that Science and Technology have an essential role in dealing with many problems in society. This of course affects their choice of higher education in topics that are far from STEM (Science, Technology, Engineering and Mathematics).

In a broader vision there is no opposition between culture and technology, as it is described by Fettweis [11]. In fact, the more recent theory of 'technological mediation' offers a framework to analyze the roles that technologies play in human existence and in society [25]. The basic idea is that technologies, when they are used, help to shape the relations between human beings and the world. Hence technological products and services should not be considered to be opposed to human subjects, or as mere extensions of human beings, they should be seen as mediators of human-world relations. So technological development is part of the cultural development of mankind. Conversely education in all branches of humanities requires also insight in technology. 


\section{CONCLUSION}

New generations of students pose new challenges to our pedagogical axioms and modes of practice. On the other hand, insights from psychology, education sciences, and brain research reveal the important role of a growth mindset. This opens avenues for new educational approaches for the fundamental concepts in circuits and systems. Rather than working with abstract definitions, the concepts should be illustrated using simple cases or inspiring geometrical arguments and their values are motivated by referring to societal problems and issues. The students gradually grasp the idea by refining the concepts. In this manner, they may make abstraction from the experiences and are more open to adopt mathematical and algebraic descriptions. A serious redesign of EE education is inevitable using techniques that meet the needs of current and prospective students. This is very much in line with many messages and achievements of Alfred Fettweis. Hence, we recommend strongly that the CAS community organizes research and didactical experimentation projects where CAS is taught with a growth mindset. This requires deep insights of the teachers and the students, and their roadblocks. A stimulating example has been recently published by Jo Boaler on the mathematical mindset for algebra. Based upon algebraic research it is experimentally shown that it is more helpful for students to learn algebra through studying pattern growth where a variable represents a case number, and can vary, before learning about "solving for x."

https://www.youcubed.org/algebra/.

We learn from Professor Fettweis that teaching circuits, signals, systems should emphasize rigor where appropriate and should avoid rigor or rigidity where unnecessary. A pragmatic approach should be taken that is a hybrid interplay between continuous time and discrete time. In the learning process students should learn from mistakes and the tests should place less emphasis on memorization of factual data, that can be easily accessed via internet.

Professor Fettweis has emphasized the societal role of engineers. He connects it directly with a moral obligation for the design engineer to fully understand and master the device or service that he/she has designed. Only in this way one can expect that a layman will have confidence in a complicated system, that controls a potentially dangerous mechanism. Hence this societal role and responsibility should be an essential element in our teaching CAS. Moreover, the attention span of the students can be enhanced by appealing and societally relevant design experiments with sound practical components and assessment methods. It should be close to societal issues and be rooted in interdisciplinary thinking and practice. All courses should include promotion of engineering ethics, systems engineering and professional conduct. It is expected that the IEEE CAS Society, and IEEE as a whole will take a leading role in this endeavor.

\section{ACKNOWLEDGMENT}

The author acknowledges stimulating discussions within the CAS Education and Outreach (CASEO) Technical Committee of IEEE CAS Society. http://ieee-cas.org/community/technicalcommittees/circuits-and-systems-education-and-outreachtechnical-committee-caseo-tc

\section{REFERENCES}

[1] N.A. Bradbury, "Attention span during lectures: 8 seconds, 10 minutes, or more?" Adv Physiol Educ. 2016 Dec 1;40(4):509-513. doi: 10.1152/advan.00109.2016.

[2] D. Barry, "The brain, technology and education "e-Teaching, Management strategies for the classroom", June 2016, http://www.acel.org.au/acel/ACEL_docs/Publications/e-Teaching/2016/eTeaching 2016 19.pdf

[3] Y. Tsividis, "Some thoughts on introducing today's students to electrical engineering," IEEE CAS Newsletter, vol. 9, no. 1, p. 1, 6-7, Mar. 1998.

[4] Y. Tsividis, "Turning students on to circuits," IEEE Solid-State Circuits Newsletter, vol. 13, no. 1, pp. 6-9, Jan. 2008.

[5] C.W. Wuh, "From the editor," IEEE CAS Magazine, February 2018, p. 3.

[6] J. Boaler, "Mathematical mindsets: Unleashing students' potential through creative math, inspiring messages and innovative teaching", Jossey-Bass, Wiley, 2016.

[7] R.K. Anderson, J. Boaler, and J. A. Dieckmann, "Achieving Elusive Teacher Change through Challenging Myths about Learning: A Blended Approach", Educ. Sci. 2018, 8(3), 98; https://doi.org/10.3390/educsci8030098

[8] https://www.youcubed.org/mindset-boosting-videos/

[9] A. Fettweis, an oral history conducted in 1997 by Frederik Nebeker, IEEE History Center, Hoboken, NJ, USA. http://ethw.org/OralHistory:Alfred_Fettweis

[10] A. Fettweis, Theory of Resonant-Transfer Circuits, in "Network and Switching Theory," Giuseppe Biorci (Ed.), Academic Press in 1968.

[11] A. Fettweis, "Engineering science and education: requirements imposed by modern technology and society", IEE Engineering Science and Education Journal, vol. 1, No. 5, pp. 216-220, October 1992.

[12] S. Mahajan, "Street-fighting mathematics, the art of educated guessing and opportunistic problem solving", MIT Press, March 2010. http://mitpress.mit.edu/books/street-fighting-mathematics

[13] J. Vandewalle, "Short cuts in circuits and systems education. With a case study of the Thévenin/Helmholtz and Norton/Mayer equivalents", Proc. IEEE International Symposium on Circuits and Systems (ISCAS), Seoul, Korea, May 2012, pp. 2925-2928.

[14] B. Ayazifar, "Can we make signals and systems intelligible, interesting and relevant?", IEEE CAS Magazine, vol. 9. No. 1, Q1 2009, pp. 48-58.

[15] B. Ayazifar, "The elegant geometry of Fourier analysis," Proc. IEEE International Conference on Circuits and Systems (ISCAS), Seoul, Korea, May 2012.

[16] V. Oklobdzija, "In Memoriam, Alfred Fettweis", IEEE CAS Newsletter Volume 9, Issue 5, October 2015.

[17] P. Dewilde, “The power of orthogonal filtering," IEEE CAS Magazine, xx 2018, p. xx.

[18] National Academy of Engineering, "Grand challenges of engineering" http://www.engineeringchallenges.org/.

[19] V. Oklobdzija, "President's message 2014: Vision", IEEE Circuits and Systems Society webpage, http://ieee-cas.org/.

[20] IEEE CAS Outreach Workshop in Lausanne 2014: Environmental Impact of Circuits and Systems, IEEE Circuits and Systems Newsletter, Vol. 8, Issue 
6, $\quad 2014 . \quad$ hecember $\quad 2$ //cassnewsletter.org/Volume8-

Issue6/CAS_Outreach_News.html

[21] General data Protection Regulation, (GDPR) Regulation EU 2016/679

https://www.eugdpr.org

https://ec.europa.eu/commission/priorities/justice-and-fundamental-

rights/data-protection/2018-reform-eu-data-protection-rules_en

[22] Y. Berbers, M. Hildebrandt, J. Vandewalle, e.a. "Privacy in an age of the internet, social networks and Big Data", Position paper 49b, Royal Flemish Academy of Belgium for Science and the Arts, 2018.

http://www.kvab.be/sites/default/rest/blobs/1501/tw_privacy_en.pdf 